\title{
Study of Galfenol direct cytotoxicity and remote microactuation in cells
}

Carolina, Vargas-Estevez ${ }^{1 *}$, Andreu Blanquer ${ }^{2}$, Prabesh Dulal ${ }^{3}$, Rafael. P. del Real ${ }^{4},{\text { Marta } \text { Duch }^{1} \text {, Elena Ibañez }}^{2}$, Leonardo Barrios ${ }^{2}$, Gonzalo Murillo ${ }^{1}$, Núria Torras ${ }^{1}$, Carme Nogués ${ }^{2 *}$, Bethanie J.H. Stadler ${ }^{5}$ José A. Plaza ${ }^{1}$, Jaume Esteve ${ }^{1}$.

1. Instituto de Microelectrónica de Barcelona, IMB-CNM (CISC), Campus UAB, Bellaterra (Barcelona), 08193, Spain.

2. Departament Biologia Cel-lular, Fisiologia i Immunologia, Facultat Biociències. Universitat Autònoma de Barcelona, Bellaterra (Barcelona), 08193, Spain.

3. Department of Chemical Engineering \& Materials Science, University of Minnesota Twin Cities, Minneapolis, MN 55455, USA.

4. Instituto de Ciencia de Materiales de Madrid (ICMM-CSIC), Madrid, 28049, Spain.

5. Department of Electrical \& Computer Engineering, University of Minnesota Twin Cities, Minneapolis, MN 55455, USA.

${ }^{+} \mathrm{CV}-\mathrm{E}$ and $\mathrm{AB}$ contributed equally to this work.

\begin{abstract}
Remote microactuators are of great interest in biology and medicine as non-invasive intracellular stimulation tools. Remote actuation can be achieved by active magnetostrictive transducers which are capable of changing shape in response to external magnetic fields thereby creating controlled displacements. Among the magnetostrictive materials, Galfenol, the multifaceted iron-based smart material, offers high magnetostriction with robust mechanical properties. In order to explore these capabilities for biomedical applications, it is necessary to study the feasibility of material miniaturization in standard fabrication processes as well as evaluate the biocompatibility. Here we develop a technology to fabricate, release, and suspend Galfenol-based microparticles, without affecting the integrity of the material. The morphology, composition and magnetic properties of the material itself are characterized, and the direct cytotoxicity of Galfenol is evaluated in vitro using human macrophages and osteosarcoma cells. In addition, cytotoxicity and actuation of Galfenol microparticles are evaluated using human macrophages. The biological parameters analyzed indicate that Galfenol is not cytotoxic, even after internalization of some of the particles by macrophages. The microparticles were remotely actuated

\footnotetext{
* Corresponding Author: carolina.vargas @ csic.es, Microelectronics Institute of Barcelona, IMB-CNM (CSIC), Campus UAB, E-08193, Bellaterra, Barcelona, Spain. Tel. + 34935947700 Ext. 2495. (Carolina Vargas-Estevez) and Carme Nogués, carme.nogues@uab.cat, Departament Biologia Cel-lular, Fisiologia i Immunologia, Facultat Biociències. Universitat Autònoma de Barcelona, Bellaterra (Barcelona), 08193, Spain.
} 
forming intra- and extracellular chains that did not impact the integrity of the cells. The results propose Galfenol as a suitable material to develop remote microactuators for cell biology studies and intracellular applications.

Keywords: Galfenol; Magnetostriction; Smart Materials; Microactuators; Biocompatibility.

\section{Introduction}

The development of biological or biomedical microelectromechanical systems (BioMEMS) has created multipurpose tools able to individually address biochemical [1] or mechanical [2] processes carried out by cells. Besides their sensing abilities, there has been a growing interest in the actuation features offered by these devices through electric [3], mechanical [4] or magnetic [5] forces. With the introduction of smart materials into these devices, it has been possible to achieve externally addressable control, avoid tethering problems, facilitate targeting, and localize actuation [6]. Among these materials, giant magnetostrictive solutions have emerged as effective tools to create remotely controlled deformations using external magnetic fields [7], to act by themselves [8] or in combination with other smart materials in tandem to create more complex capabilities [9]. In fact the integration of magnetoelastic materials into MEMS has brought an outstanding performance, as they give properties of "self-test, self-calibration and remote sensing and actuation" already stated by Gibbs [10], which certainly offers the perfect tool for biomedical less invasive tools.

One of the most versatile examples of giant magnetostrictive materials is Galfenol [11], an alloy made of iron and gallium, able to transduce magnetic energy into mechanical deformation with strains that go up to hundreds ppm at low saturating magnetic fields [12]. Its mechanical strength overcomes the mechanical problems of its famous counterpart Terfenol-D which has a higher magnetostriction ( 2000 ppm) [13], but is very brittle at room temperature [14]. On the contrary, Galfenol has high ductility and great durability under tensile, bending and compressive forces [15], which are preserved at micro- and nanoscale [16]. It can also be sputtered from a fixed composition alloy target, creating high quality and compositionally consistent thin films [17], with the correct proportion of gallium and iron to ensure its magnetostriction [17]. The versatility of this material has opened the path to multiple applications from microactuators [18] to micro- and nanorobots [19]. Although the introduction 
of Galfenol to BioMEMs is only exploratory to date [20], it still is necessary to ensure its biocompatibility, as a first step in the development of medical applications. The first attempt to evaluate the biocompatibility of Galfenol [21] was performed through indirect cytotoxicity analyses using millimetric blocks in contact with the culture media. Once the blocks were removed, the media was used for fibroblast incubation with no adverse results in the cell survival. Additionally the biodegradable properties of Galfenol were also found to be negligible [21], showing a great future for the alloy in bioapplications. Going down in scale, Galfenol nanowires were also internalized by cells, showing cell viability in a preliminary qualitative toxicity assay [22].

The present study evaluates the integration of Galfenol to suspended microdevices for cell biology studies and intracellular applications. As a first stage, thin films of the alloy were fabricated to characterize the properties of the material and to perform an initial in vitro test, growing cells (human macrophages and osteosarcoma cells) directly on the Galfenol surface. Then, to evaluate the reaction of macrophages to the internalization of Galfenol, $3 \mu \mathrm{m} \times 3 \mu \mathrm{m} \times 1 \mu \mathrm{m}$ silicon oxide microparticles were fabricated, covered with a thin layer of the alloy, and added to cell cultures. Finally, by means of an external magnetic field, the interactions between actuated microparticles and cells were analyzed.

\section{Materials and Methods}

\subsection{Fabrication of Galfenol test surfaces and Galfenol-based microparticles}

Uniform Galfenol films, $160 \mathrm{~nm}$ thick, were deposited by radio frequency (RF) magnetron sputter deposition onto polysilicon substrates. The parameters of the deposition were $200 \mathrm{~W}$ for $45 \mathrm{~min}$ using a target with 18.4 at $\% \mathrm{Ga}$ nominal (Etrema Inc., USA) and an Ar pressure of 2.8 mTorr (30 sccm).

Galfenol-based microparticles were fabricated using $1 \mu \mathrm{m}$ thick thermal silicon oxide grown on a $100 \mathrm{~mm} \emptyset \mathrm{p}$ type silicon wafer (Fig. 1A). Positive UV photoresist was used to define an array of $3 \mu \mathrm{m} \times 3 \mu \mathrm{m}$ squares separated by $3 \mu \mathrm{m}$ (Fig. 1B). The oxide was patterned by Reactive Ion Etching (RIE; Alcatel 601E, France) with $\mathrm{CHF}_{3}$ plasma for $11 \mathrm{~min}$ (Fig. 1C), followed by a strip of the photoresist, leaving the silicon oxide particles (Fig. 1D). Next, partial isotropic etching by RIE created a narrow anchor (Fig. 1E), followed by a thin layer deposition of Galfenol $(160 \mathrm{~nm})$ by same process used for the films above (Fig. 1F). The particles were released using 
mechanical peeling [1] which broke the silicon anchor without affecting the integrity of the Galfenol film (Fig. 1 G). Finally the particles were collected and suspended in ethanol for their storage (Fig. 1H).

A

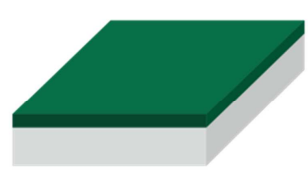

E

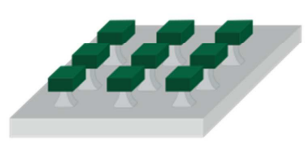

Silicon
B

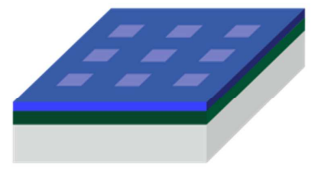

$\mathbf{F}$

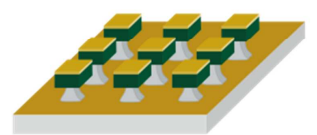

Photoresist
C

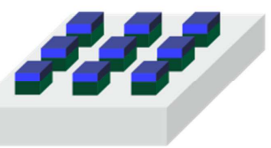

G

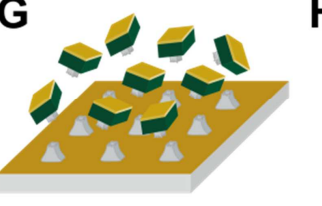

Galfenol
D

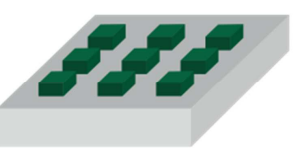

H

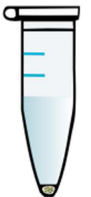

Fig.1. Fabrication process of Galfenol microparticles. (A) Fabrication started with a silicon substrate with $1 \mu$ m thick thermal oxide growth. (B)(C) (D) Photolithography followed by a vertical etching defined the structure. (E) Anchors were made by etching the silicon isotropically. (F) $160 \mathrm{~nm}$ of Galfenol was deposited by sputtering. (G) The particles were mechanically released, (H) collected and suspended in ethanol.

Finally, another thin layer of Galfenol $(160 \mathrm{~nm})$ was sputtered on glass coverslips (Gold Seal, Portsmouth, NH, USA) with dimensions of $18 \mathrm{~mm} \times 18 \mathrm{~mm} \times 0.13 \mathrm{~mm}$ for magnetostriction measurements.

\subsection{Characterization of the alloy surfaces and the microparticles}

Grain morphology of the samples was observed using a scanning electron microscope (SEM; Carl Zeiss, Auriga Series, $3 \mathrm{KV}$, Germany). The surface roughness of both samples was measured by means of an atomic force microscope (AFM; Veeco, USA) using the tapping mode. The morphology of the microparticles was observed using SEM, in order to verify the shape, size and anchor for releasing them effectively. A microparticle was cross sectioned by focused ion beam (FIB; Leo 1530 Zeiss, Germany) and observed with a Gemini SEM column, to observe the interface of the Galfenol film and the microparticle. The FIB used a $\mathrm{Ga}^{+}$ion beam at an accelerating voltage of $30 \mathrm{kV}$ and a beam current of 30-100 pA. The composition of the Galfenol was confirmed through energy dispersive X-ray spectroscopy (EDX; Carl Zeiss, Auriga Series, $3 \mathrm{KV}$, Germany). The crystalline microstructure of the alloy was studied using an X-ray diffractometer (XRD; Bruker, AXS D8-Advance), for 
which the thin film specimens were cut into rectangular pieces $(0.8 \mathrm{~cm} \times 0.7 \mathrm{~cm})$. The integrity of the microparticles after their release was evaluated by releasing a $2 \mu \mathrm{L}$ drop of the ethanol suspension onto a clean $\mathrm{Si}$ substrate. Once the ethanol evaporated, the particles were observed using SEM. In terms of functional properties of the material, the magnetic properties of the samples were measured with a vibrating sample magnetometer (VSM; MicroMag 3900; Princeton Measurements Corporation, USA) at room temperature (RT), applying a maximum field of $10 \mathrm{kOe}$. The VSM samples were $160 \mathrm{~nm}$ Galfenol films on polysilicon substrates $(6 \mathrm{~mm} \times 8$ $\mathrm{mm}$ ) and a platform of the same size with unreleased $\mathrm{Galfenol} / \mathrm{SiO}_{2}$ microparticles. Furthermore, the magnetostriction of Galfenol on glass coverslips was measured using a capacitive bridge system described in detail in [23].

\subsection{Cell lines}

Two different human cell lines were used to test the effects of Galfenol films and microparticles. THP-1 monocyte cells were grown under standard conditions $\left(37{ }^{\circ} \mathrm{C}\right.$ and $5 \% \mathrm{CO}_{2}$ ) in RPMI 1670 medium (Life Technologies, UK) supplemented with $25 \%$ fetal bovine serum (FBS; Life Technologies, UK) and 5\% L-

glutamine (Biowest, USA). To differentiate monocytes into macrophages, $10^{5}$ cells were seeded into 4-well plates and treated with $0.16 \mathrm{mM}$ phorbol-12-myristate-13-acetate (PMA; Sigma, USA) for $48 \mathrm{~h}$. The human osteosarcoma cell line Saos-2 (ATCC, USA) was cultured in Dulbecco's modified Eagle medium (Invitrogen, USA) with $10 \%$ FBS under standard conditions.

\subsection{Cell viability assay}

Two different cell viability assays were conducted to measure direct cytotoxicity. In the first study, the cells were seeded onto $1 \mathrm{~cm} \times 1 \mathrm{~cm}$ Galfenol-coated polysilicon films which were cleaned with absolute ethanol and sterilized with UV light for at least $1 \mathrm{~h}$ inside 4 -well culture plates. Once sterilized, $5 \times 10^{4}$ osteoblasts or $1 \times 10^{5}$ monocytes were seeded on top of each film and cultured for $24 \mathrm{~h}$ or $48 \mathrm{~h}$, respectively. In parallel, control cells were seeded directly onto glass coverslips in the absence of the Galfenol films. Once seeded, monocytes were induced to differentiate into macrophages as described above. In the second study, the effect of Galfenol microparticles $(3 \mu \mathrm{m} \times 3 \mu \mathrm{m} \times 1 \mu \mathrm{m})$ on cell viability was studied. Microparticles were added directly to differentiated macrophages at a 1:1 ratio and incubated for $24 \mathrm{~h}$. In both assays, cell viability was evaluated by 
detecting the activity of intracellular esterases using the LIVE-DEAD® Viability/Cytotoxicity kit for mammalian cells (Invitrogen, USA), according to the manufacturer's protocol. Images from different regions of the cell cultures were captured using an inverted epifluorescence microscope (Olympus IX7, Germany). Each experiment was done in triplicate. A minimum of 300 cells were analyzed per group. Data were analyzed for significance using the Fisher's exact test for comparison between groups. Statistical significance was considered when $\mathrm{p}<$ 0.05 .

\subsection{Cell morphology analysis}

The same samples used for the cell viability assays were subsequently processed for SEM morphological analysis. Briefly, cultured cells were rinsed twice in phosphate buffered saline (PBS), fixed in $4 \%$ paraformaldehyde (Sigma, USA) in PBS for 15 min at RT and rinsed twice in PBS. Cell dehydration was performed in series of ethanol (50\%, 70\% and 90\% once, and twice in 100\%), 7 min each. Finally, samples were dried using hexamethyldisilazane (HMDS; Electron Microscopy Sciences, USA) for 15 min, mounted on special stubs and analyzed using SEM.

\subsection{Internalization assay of Galfenol-based microparticles}

The uptake of Galfenol-based microparticles by macrophages was analyzed by confocal scanning laser microscopy (CLSM; Leica TCS SP5, Germany) and SEM. For this study, microparticles were added to differentiated macrophages cultured on glass coverslips at a 1:1 ratio ( $10^{5}$ microparticles: $10^{5}$ macrophages $)$. After $24 \mathrm{~h}$, cells were washed with a medium without serum and stained with Cell Mask Deep Red plasma membrane stain (Life Technologies, UK) for $10 \mathrm{~min}$ at $37^{\circ} \mathrm{C}$. Coverslips were placed upside-down on specific bottom glass dishes (MatTek, USA). Samples were visualized using a 63x oil immersion objective, and xyz sequential acquisition was performed to determine the location of the microparticles within the cell. For SEM images, cells were fixed, dehydrated and dried as discussed earlier in cell morphology analysis (section 2.5). In order to confirm the uptake of the microparticles inside the cells, FIB was used to mill the cell and localize the microparticle in a cross sectional view. The ion beam was operated with an acceleration voltage of $30 \mathrm{kV}$ with varying beam current ranging from $2 \mathrm{nA}$ to $500 \mathrm{pA}$. The cross-sectional milled area was analyzed by SEM.

\subsection{In vitro magnetic evaluation of the Galfenol microparticles}


To asses if a magnetic field produced any effect in the macrophages, with or without internalized Galfenol microparticles, $10^{5}$ macrophages were incubated for $24 \mathrm{~h}$ with $10^{5}$ Galfenol microparticles on reticulated coverslips. Then, an external magnetic field was applied through a cylindrical neodymium permanent magnet of $20 \mathrm{~mm}$ diameter and $10 \mathrm{~mm}$ height (S-20-10-N, Supermagnete, Germany) which was positioned between the 4 wells, with the north face parallel to the base of the plate. This magnet had a magnetic flux density on the edge of 250 Gauss at the edge of the plate $(20 \mathrm{~mm}$, which corresponds to the center of each of the wells. This created a magnetic field gradient, with a higher intensity in the cells located close to the border of the culture plate. Images of different areas of the cell culture were taken under an inverted microscope, before and after 1 min of the application of the magnetic field. Cell cultures were maintained for another $24 \mathrm{~h}$ in the presence of the magnet under standard conditions. Afterward, microtubules distribution was evaluated by immunofluorescence. Cells with microparticles and control cells were fixed in $4 \%$ paraformaldehyde in PBS for $15 \mathrm{~min}$ at RT to evaluate microtubules distribution by immunofluorescence. Cells were permeabilized with $0.1 \%$ Triton X-100 (Sigma, USA) in PBS for 15 min and blocked for 25 min with $1 \%$ bovine serum albumin (BSA; Sigma, USA) in PBS at RT. Samples were then incubated with a mouse anti- $\alpha$-tubulin primary antibody (1:1000; Sigma, USA) for 60 min at RT and washed with $1 \%$ BSA-PBS. Next, the samples were incubated with a FITC-conjugated anti-mouse IgG (1:150; Sigma, USA) for 50 min at RT, washed with $1 \%$ BSA-PBS, and incubated again with Hoechst 33258 (Sigma, USA). Finally, the samples were washed in 1\% BSA-PBS, air-dried and mounted on MatTek dishes using Fluoroprep mounting solution (Biomerieux, France). The samples were evaluated with a CLSM, where a series of horizontal optical sections were collected. Orthogonal projections were generated with Imaris software (Bitplane AG, Switzerland). 


\section{Results and Discussion}

\subsection{Morphological and structural studies of the alloy (SEM-FIB)}

Surface topography is one of the most significant factors when the biocompatibility of a material is evaluated, as this is the first interaction that defines the cell attachment and further survival of the cells [24] [25]. The roughness of the Galfenol alloy was studied by observing the grain structure over the samples. The grain morphology of the alloy on polysilicon revealed large grains (Fig. 2A left) that are typical for this type of substrate. Observing these grains in detail, the AFM results revealed that there was a bimodal grain size distribution: larger grains of $203 \pm 11 \mathrm{~nm}$ (Fig. 2A middle) of polysilicon coated with smaller grains of $36 \pm 4 \mathrm{~nm}$ Galfenol (Fig. 2A right). In the case of the alloy sputtered over smooth silicon oxide microparticles, only the small uniform grains of Galfenol were observed in SEM (Fig 2B left). AFM of the microparticles indicate that Galfenol on silicon oxide was not very rough with monomodal grain size of $32 \pm 4 \mathrm{~nm}$ (Fig. 2B middle-right). The phase image of the samples showed that the adhesion of these grains to the substrate was uniform. These results are encouraging because a smooth surface is likely to promote interfacial biocompatibility and enhance the early adhesion of the cells [26].

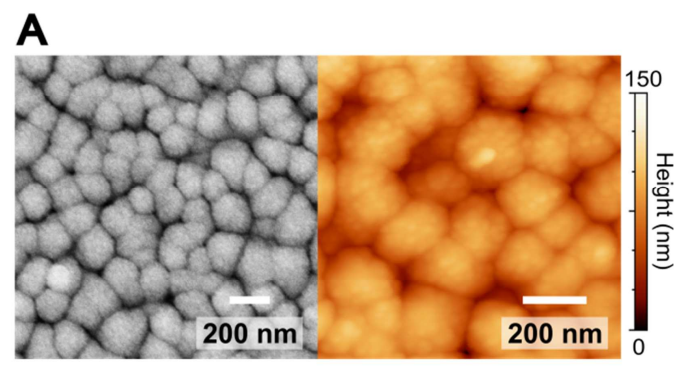

B

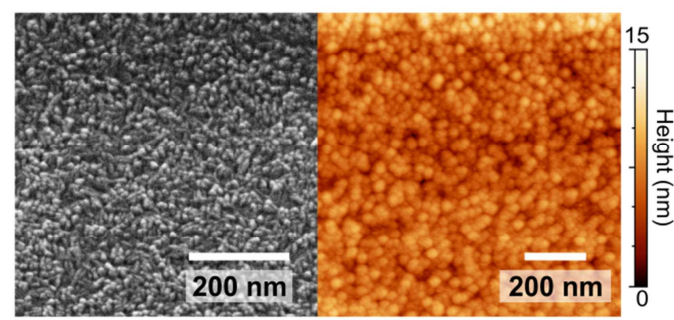

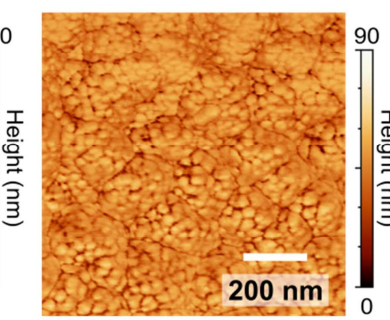

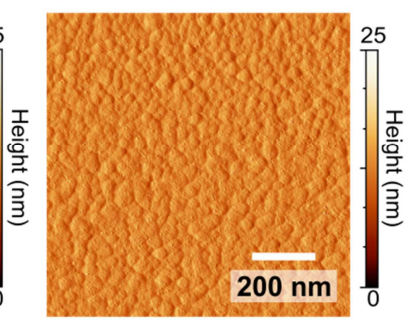

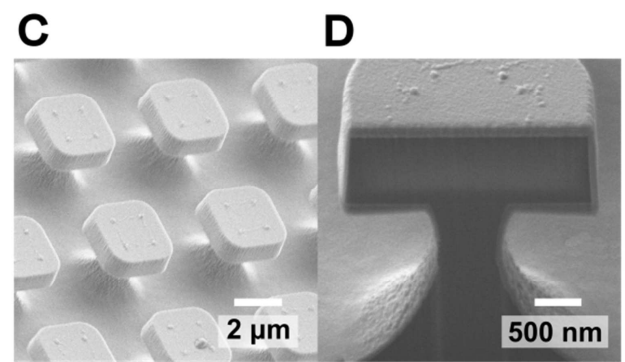

E $\quad F$

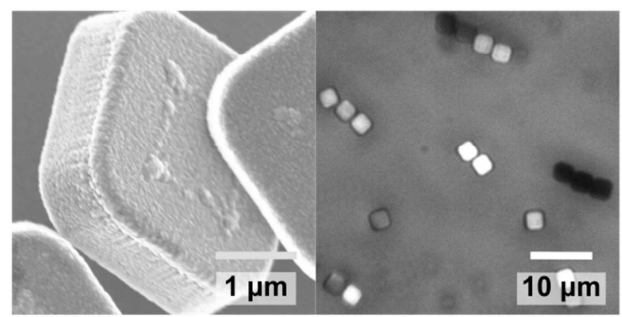

Fig. 2. Morphology analysis of the Galfenol alloy. Grain observed of Galfenol over polysilicon by SEM image (A left), by AFM topography image (A middle) and by AFM phase image (A right) of the same polysilicon sample, giving more detail of the inner smaller grains (AFM scan size: $1 \times 1 \mu \mathrm{m}^{2}$ ). The grain of Galfenol observed over the silicon oxide microparticles by SEM image (B left), by AFM topography image (B middle) and by AFM phase image (B right) of the same microparticle (AFM scan size: $500 \times 500 \mathrm{~nm}^{2}$ ). SEM images of $(\mathrm{C})$ the Galfenol-based microparticles attached to the silicon substrate, (D) cross section of the microparticle milled by FIB, (E) microparticles released and (F) the same released microparticles after the exposure of a magnetic field, showing chain alignment. 
Continuing with the morphology analysis, the silicon oxide microparticles observed by SEM (Fig. 2C-F), revealed a well-defined shape. The Galfenol layer was uniform all over the surface of the particles, by the cross-sectional FIB image of the microparticle in Fig. 2D. The thickness of the Galfenol layer observed through this cross section over the particle was $156 \mathrm{~nm}$ and the anchor's width was $957 \mathrm{~nm}$, which compared to the dimensions of the microparticle $(3 \mu \mathrm{m} \times 3 \mu \mathrm{m} \times 1 \mu \mathrm{m})$ made it easy to peel the microparticles off of the substrate. This clean release method was very effective, ensuring a density of around $1.5 \times 10^{6}$ microparticles per $1 \mathrm{ml}$ of ethanol. Additionally, this method also preserved the integrity of the alloy (Fig. 2E) and prevented the contamination with external chemicals that are often present in other release processes, such as chemical etching, and that nowadays are avoided due to toxicity [27]. Once in suspension, the microparticles were exposed to a magnetic field to observe their behavior and determine if they would cluster. Under an optical microscope, it was possible to observe chains of microparticles as a result of dipolar interactions (Fig. 2F). These dipolar interactions orient the microparticles into chains with magnetizations parallel to the applied magnetic field.

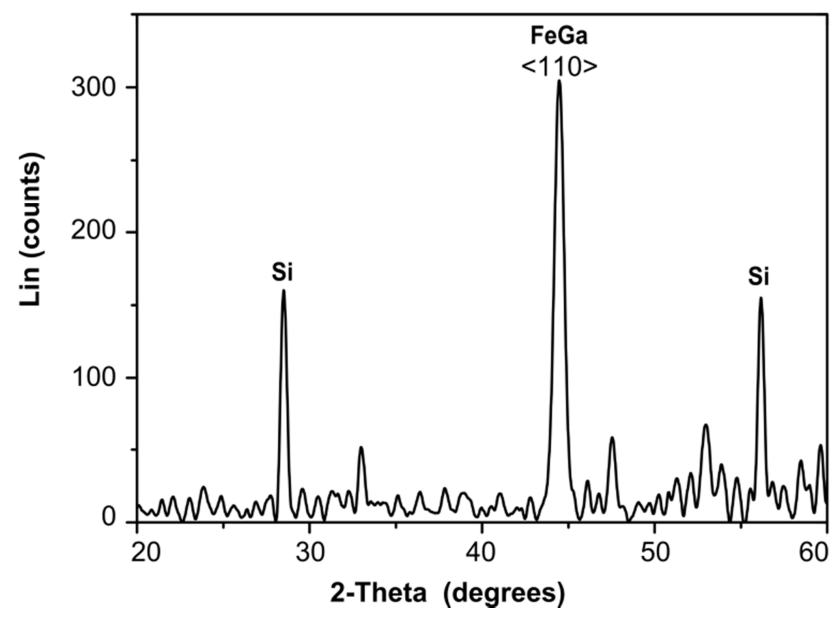

Fig. 3. X-Ray analysis of the Galfenol film. The fundamental $\left(\begin{array}{lll}1 & 1 & 0\end{array}\right)$ reflection of the magnetostrictive alloy is indicated. Other peaks correspond to the silicon (100) wafer.

The composition of the Galfenol alloy was measured using EDX to be $15.63 \% \mathrm{Ga}$ and $84.37 \% \mathrm{Fe}$ (atomic \%) in both samples, maintaining the target stoichiometry which as the best magnetostrictive coefficients of the alloy system. Additionally, the theta -2 theta XRD pattern showed a peak at $44.4^{\circ}$ that corresponds to the tendency of the material to be oriented along the (110) orientation (Fig. 3) which is the closest packing of the BCC structure. 
A

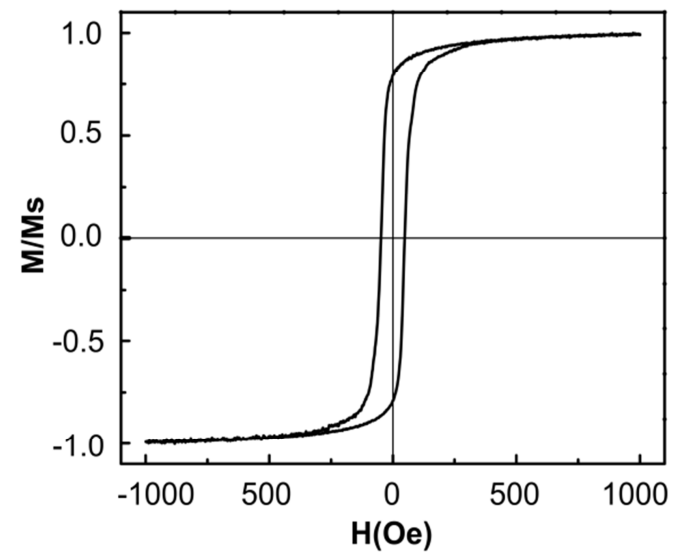

B

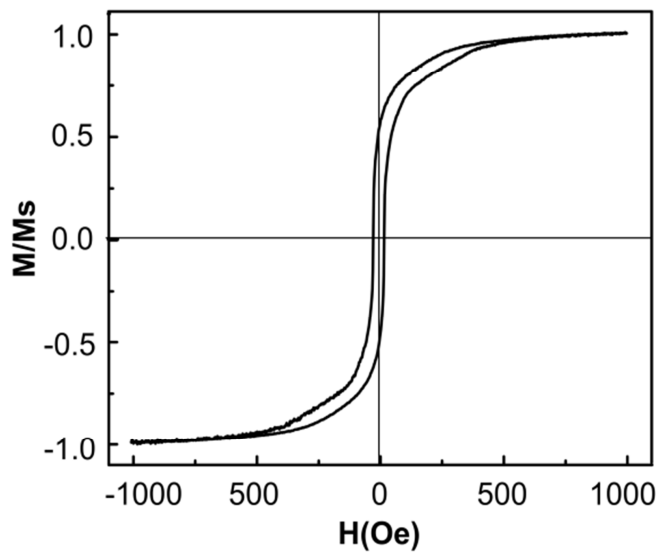

Fig. 4. Normalized hysteresis loops at room temperature of (A) Galfenol over polysilicon film and (B) the Galfenol-based microparticles still attached to the platform

The hysteresis loops measured by VSM revealed a higher coercivity for the films on polysilicon (Fig. 4.A) in contrast to the microparticles when they were still attached to the chip (Fig. 4.B). The coercivity of the Galfenol film on the structured polysilicon sample measured was 48.98 Oe and in the microparticles (still attached) was 21.11 Oe. These low values of coercivity suggested a soft magnetic behavior of Galfenol in thin films, a behavior consistent with the results found in [28]. Furthermore, a magnetostriction constant of approximately 90 ppm was measured on the $160 \mathrm{~nm}$ thick films deposited over coverslips following the capacitance bridge method stated in [23].

\subsection{Cytotoxicity in the Galfenol test surfaces}

The cytotoxicity of Galfenol films was analyzed by quantifying the number of live cells with esterase activity (presenting green fluorescence) and the number of dead cells (presenting red fluorescence) (Fig.5A). Galfenol films had no effect on the viability of either monocytes differentiated into macrophages or Saos-2 cells. The percentage of live cells was higher than $93 \%$ in both cell lines, and no significant differences were observed when compared with control cells grown on glass coverslips (Fig.5B). 

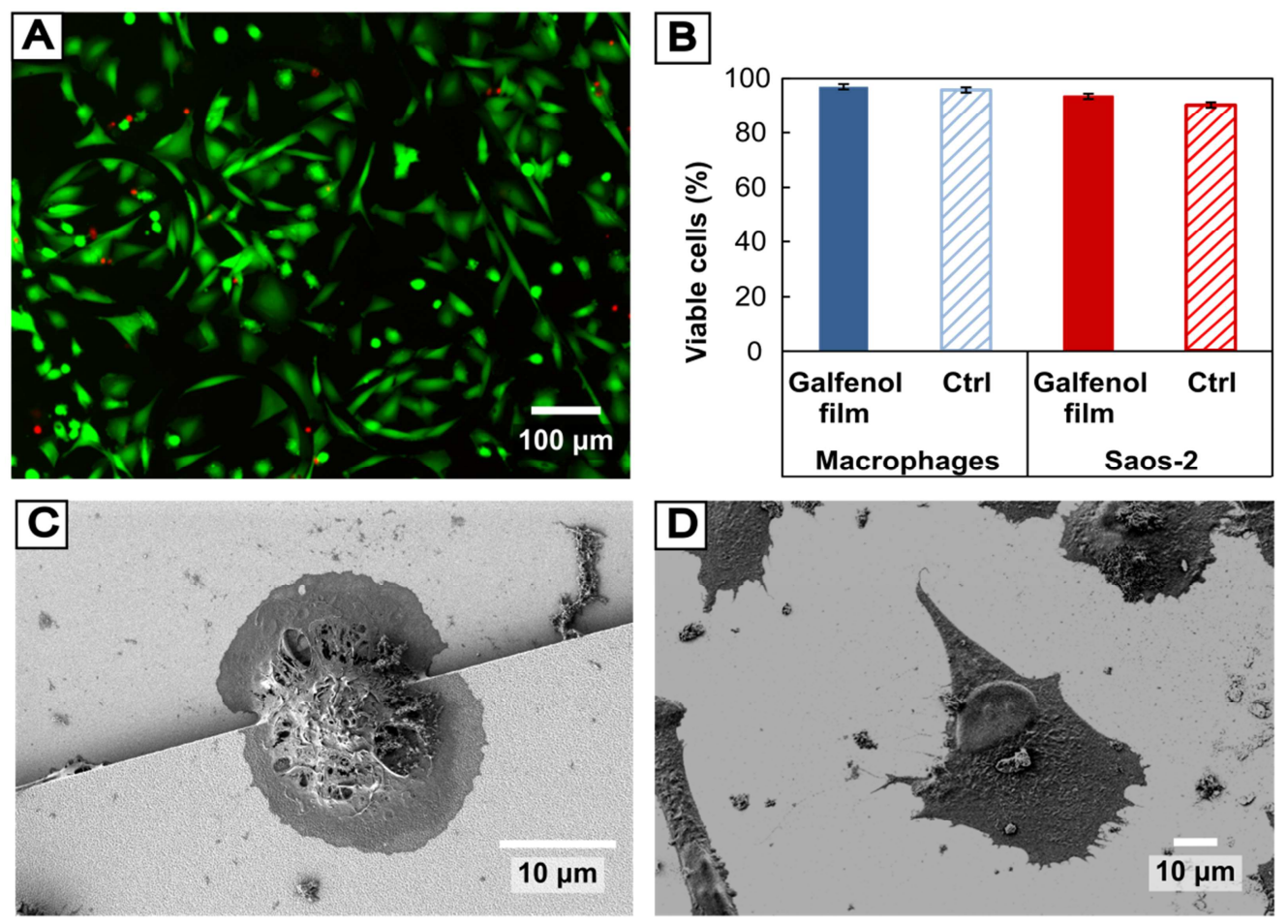

Fig. 5. Viability and morphology of macrophages and Saos-2 cells growing on Galfenol films. (A) Live (green) and dead (red) Saos-2 cells and (B) percentage of viable macrophages and Saos-2 cells growing on top of Galfenol films or glass coverslips (Ctrl), evaluated using the LIVE/DEAD viability/cytotoxicity kit. SEM images of well-spread macrophages (C) and Saos-2 cells (D) adhered to the Galfenol films.

Cell morphology was evaluated using SEM. Macrophages were completely spread and adhered onto the film surface after $48 \mathrm{~h}$ of culture (Fig. 5C). Similarly, Saos-2 cells were also well-spread and adhered to the surface, showing some cytoplasmic extensions after $24 \mathrm{~h}$ of culture (Fig. 5D). For both cell types, morphology was similar to that of control cells growing on glass coverslips. Thus, the high percentages of living cells and their normal morphology revealed that Galfenol did not affect cellular viability.

Previous studies had indirectly demonstrated the biocompatibility of Galfenol by culturing cells in conditioned medium earlier incubated with Galfenol samples for different time-points [21]. To the best of our knowledge, this is the first time that Galfenol cytotoxicity has been tested directly by culturing the cells over surfaces of this alloy. 


\subsection{Cell internalization of the Galfenol-based microparticles}
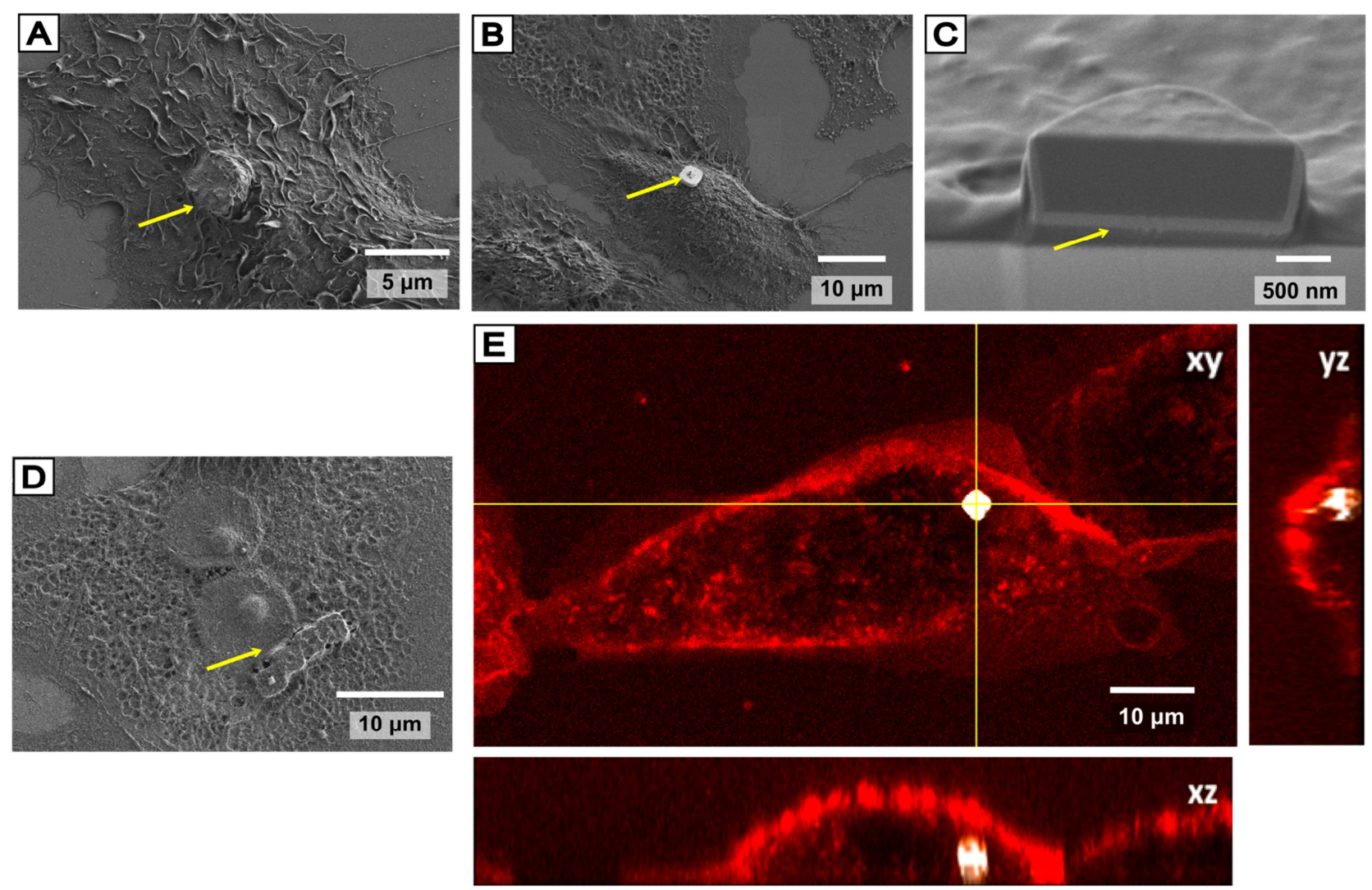

Fig. 6. Galfenol microparticles internalization. SEM images of macrophages with (A) an internalized microparticle (arrow) and (B) a microparticle on the plasma membrane (arrow). (C) Detail of a FIB-milled macrophage with an internalized microparticle. The microparticle, with the Galfenol surface (arrow), is surrounded by the plasma membrane. (D) SEM image with several internalized microparticles. (E) CLSM orthogonal image of macrophage with one internalized microparticle.

When Galfenol microparticles were added to the cell cultures, the percentage of live macrophages was $87.7 \%$ after $24 \mathrm{~h}$ in culture and no significant differences were observed compared with macrophages cultured in absence of microparticles $(89.8 \%)$.

Microparticle internalization was evaluated using SEM, FIB and CLSM. SEM images showed some macrophages with Galfenol a microparticle internalized (Fig. 6A) and others with a microparticle on its plasma membrane (Fig. 6B). Some macrophages previously selected by SEM were cross-sectioned using the FIB to confirm that microparticles were certainly inside the cells, and not under them, as it can be clearly seen in Fig. 6C. No differences in cell morphology were observed when compared with control macrophages cultured in the absence 
of microparticles. In all cases, cells showed rounded or polygonal shape, with a nucleus containing several nucleoli, a sign of cellular activity, also observed in macrophages that had more than one internalized microparticle (Fig. 6D). Furthermore, microparticle internalization was also confirmed by CLSM (Fig. 6E), in which cells can be analyzed alive, without fixation and dehydration. When labeling the plasma membrane, CLSM analysis allowed to determine precisely whether microparticles were located inside or outside the cells. Previous studies had demonstrated that macrophages can phagocyte different artificial microstructures such as polysilicon, gold or chromium microparticles (3 $\mu \mathrm{m}$ in diameter) without effects on viability [29] [30]. Our results showed many macrophages with one or more microparticles in their cytoplasm and, together with the viability, SEM and FIB results, they demonstrated that Galfenol internalization did not change any of the biocompatibility parameters analyzed. .

\subsection{Magnetic effect on the Galfenol-based microparticles}

A magnetic field was applied to observe the remote actuation possibilities for extra- and intra-cellular Galfenol microparticles. Before the application of a magnetic field, microparticles were scattered throughout the culture plate (Fig. 7A, yellow arrows) and the cytoplasm of some cells. However, after the field was applied, a similar chaining effect as that seen in the ethanol suspension was observed. After $1 \mathrm{~min}$, almost all isolated microparticles had joined a chain, bonded either to the microparticles on cell surfaces or to microparticles that were apparently internalized (Fig. 7B, red arrows). In some cases, internalized microparticles gathered together in chains inside the cells upon the application of the field (Fig. 7A and B, and inset). The parameters of actuation did not cause any visible effect on the cells. This low field guaranteed that even after internalization the particles could gather together without disrupting the membrane. 

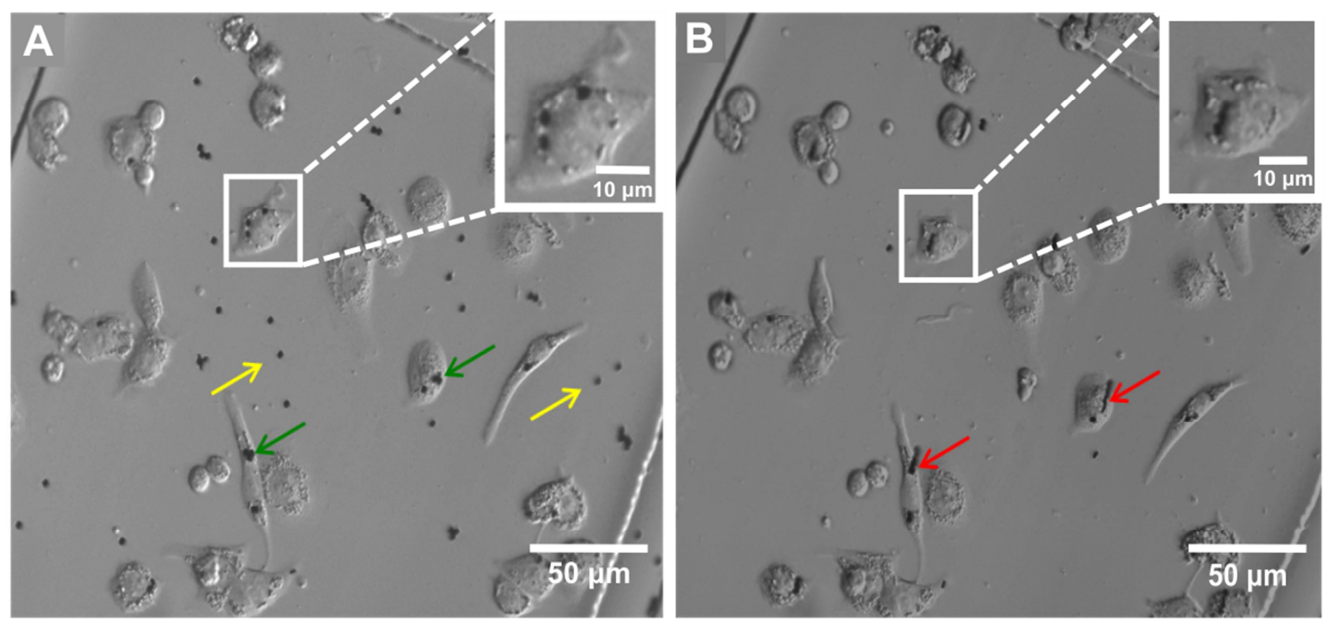

Fig. 7. Magnetic field effect on macrophage cultures incubated with Galfenol microparticles. Images captured before (A) and 1 min after (B) the magnetic field application. Free Galfenol microparticles (yellow arrows) became associated with microparticles adhered to the cell surface (green arrows), forming small chains (red arrows), after magnetic field exposure. In cells with apparently internalized microparticles (inset in A), these ones gathered together after magnetic field exposure forming small intracellular chains (inset in B).

The formation of chains among magnetic particles is a well-known effect that occurs due to dipole-dipole interactions, mostly in nanoparticles [31]. Interestingly, the chain effect observed in the Galfenol microparticles can be compared to the chains found in the magnetotactic bacteria, where the alignment allows the maximization of magnetization as this makes them more susceptible to an external magnetic field [32]. In the case of the bacteria, this chain effect is only possible through biological structures that prevent them from forming clusters whereas in this work, the remanent polarization in the microparticles aligned the domains with the magnetic field, allowing the effect even with some of the particles that were internalized in the cell. Hence, these chain structures can be seen as assembled microactuators [33] [34] or sensors with an enlarged cluster effect [35] over the cells. The integrity of the morphology in cells interacting with the microparticles was observed after $24 \mathrm{~h}$ of magnetic field exposure. Microtubules (cytoskeleton) were labeled to analyze their organization inside the cell and indirectly, the location of the microparticles. Fig. 8 shows a macrophage with several Galfenol microparticles, outside and inside the cell, with clearly defined microtubules extended throughout the cytoplasm. The presence of two microparticles inside the cell did not alter the microtubule gross distribution as it is clearly seen in Fig. 8B. Thus, it is clear that a weak magnetic field can remotely activate a particular movement of these microparticles without inducing the disruption of the cytoskeleton, nor on the viability of the cell itself. 

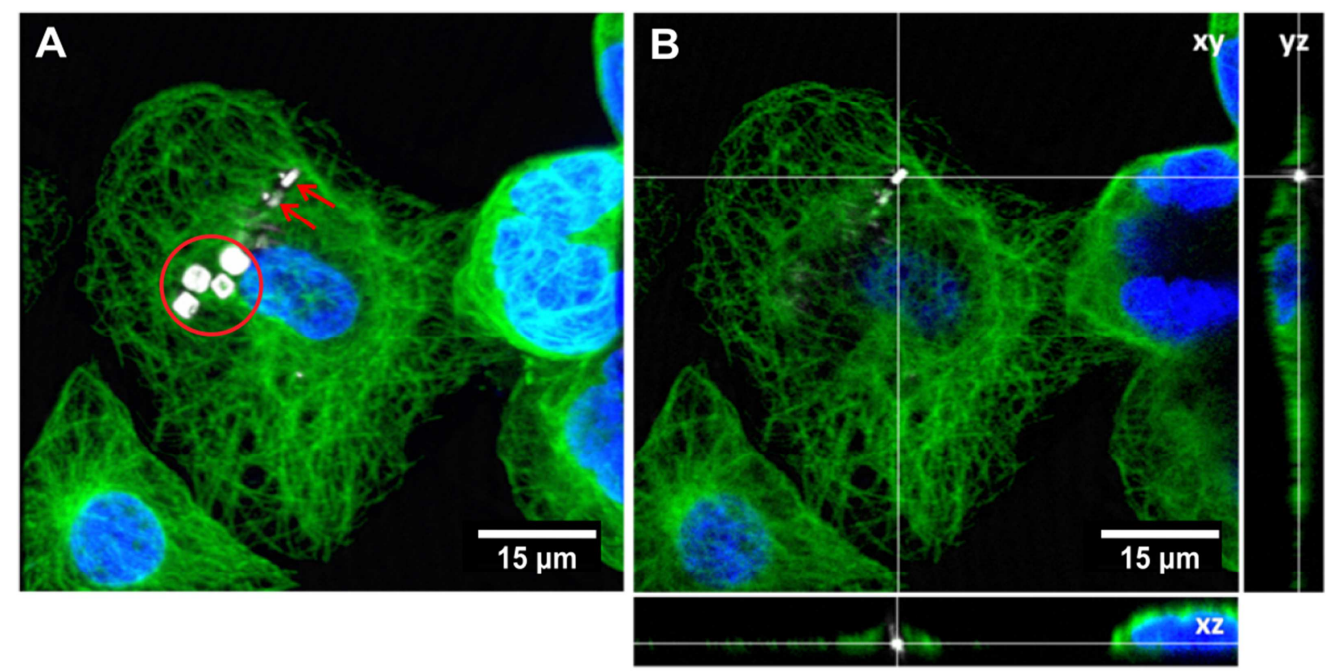

Fig. 8. Macrophage cytoskeleton interaction with Galfenol-base microparticles after magnetic exposure. (A) CLSM reconstructed $3 \mathrm{D}$ and (B) orthogonal image of the same macrophages exposed to the magnetic field $(1 \mathrm{~min})$ and cultured for $24 \mathrm{~h}$. One of the macrophages showed 6 Galfenol microparticles, 4 inside the red circle and 2 pointed by red arrows (A). Orthogonal projection (B) shows that at least two of these microparticles were inside the cell (yz image (right) and zx image (bottom)). Microtubules stained in green show a normal distribution. Nuclei stained with Hoechst are in blue and microparticles visualized by reflection are seen in white.

\section{Conclusions}

Suspended Galfenol microparticles were successfully produced through semiconductor technology processes. The alloy features were studied to guarantee that the process followed during the fabrication of the microparticles did not alter their properties, which makes this microfabrication process suitable to integrate this alloy to more complex devices. The microparticles were released with an efficient peel-off method that preserved the integrity of the material, leaving them in suspension ready for biological testing.

On the other hand, the evaluation of the biocompatibility of the alloy in vitro by analyzing the direct cytotoxicity, with films and with microparticles, on two different cell lines (osteoblasts and macrophages), demonstrated that the alloy was not cytotoxic, even after internalization of the microparticles. The cell culture with the microparticles was also exposed to a magnetic field of a permanent magnet, which in turn created chain clusters of microparticles over the cell membrane and inside the cells. These chains were remotely controlled with the magnetic vector field, performing a smooth assembled actuation that did not affect the morphology of the cells in 
the short term. Moreover, the same culture was left under the effect of that magnetic field for $24 \mathrm{~h}$ to analyze changes in the internal structure, but there were no disturbances in the microtubule gross distribution. In summary, the direct cytotoxicity and actuation in vitro tests carried out revealed that Galfenol has a good shortterm biological safety under the chosen test conditions. These results open a window for Galfenol in the development of novel integrated remote micro- and nanodevices that might include other smart materials for more complex tasks, which would include sensing and actuating inside living cells in a minimally invasive way.

\section{Acknowledgements}

This study was supported by the Spanish Government through the project MINAHE 5 (No. TEC2014-51940-C1) and ENVBIOPORAL (No. MAT2014-57960-C3-3-R), the predoctoral FPI grant (No. BES-2012-052105), the NANONEURO project PIE201350E110 (CSIC) and the Generalitat de Catalunya (2014-SGR-524). A.B. was supported by a predoctoral grant from the Universitat Autònoma de Barcelona. We wish to thank as well the IMB-CNM Clean Room (Barcelona, Spain), Prof. William P. Robbins of University of Minnesota (Minneapolis, USA) for his support to perform the magnetostrictive measurements and the Institute for Rock Magnetism (Minneapolis, USA) for its facilities.

\section{References}

[1] N. Torras, J. P. Agusil, P. Vázquez, M. Duch, A. M. Hernández-Pinto, J. Samitier, E. J. de la Rosa, J. Esteve, T. Suárez, L. Pérez-García and J. A. Plaza, "Suspended Planar-Array Chips for Molecular Multiplexing at the Microscale," Advanced Materials, vol. 28, p. 1449-1454, 2016.

[2] R. Gómez-Martínez, A. M. Hernández-Pinto, M. Duch, P. Vázquez, Z. Kirill, E. de la Rosa, J. Esteve, T. Suárez and J. Plaza, "Silicon chips detect intracellular pressure changes in living cells," Nature Nanotechnology, vol. 8, p. 517-521, 2013.

[3] X. Sui, J. Sun, L. Li, C. Zhou, X. Luo, N. Xia, Y. Yan, Y. Chen, Q. Ren and X. Chai, "Evaluation of a MEMS-Based Dual Metal-Layer Thin-Film Microelectrode Array for Suprachoroidal Electrical Stimulation," Neural Systems and Rehabilitation Engineering, IEEE Transactions on, vol. 21, no. 4, pp. 524-531, 2013.

[4] D. Desmaële, M. Boukallel and S. Régnier, "Actuation means for the mechanical stimulation of living cells via microelectromechanical systems: A critical review," Journal of Biomechanics, vol. 44, no. 8, p. 1433- 
1446, 2011.

[5] G. Bonmassar, S. W. Lee, Freeman, D. K., M. Polasek, S. I. Fried and J. T. Gale, "Microscopic magnetic stimulation of neural tissue," Nature communications, vol. 921, no. 3, 2012.

[6] D. D. Chrusch, B. W. Podaima and R. Gordon, "Cytobots: intracellular robotic micromanipulators," Canadian Conference on Electrical and Computer Engineering. IEEE CCECE 2002., vol. 3, pp. 1640-1645, 2002.

[7] M. R. J. Gibbs, E. W. Hill and P. J. Wright, "Magnetic materials for MEMS applications," J. Phys. D: Appl. Phys., vol. 37, pp. 237-244, 2004.

[8] T. Fukuda, H. Hosokai, H. Ohyama, H. Hashimoto and F. Arai, "Giant magnetostrictive alloy (GMA) applications to micro mobile robot as a micro actuator without power supply cables," Micro Electro Mechanical Systems, 1991, MEMS'91, Proceedings. An Investigation of Micro Structures, Sensors, Actuators, Machines and Robots. IEEE., pp. 210-215, 1991.

[9] A. Bayrashev, W. P. Robbins and B. Ziaie, "Low frequency wireless powering of microsystems using piezoelectric-magnetostrictive laminate composites," Sensors and Actuators A: Physical, vol. 114, no. 2, pp. 244-249, 2004.

[10] M. R. J. Gibbs, "Applications of magmems," Journal of magnetism and Magnetic materials, vol. 290, pp. 1298-1303, 2005.

[11] R. R. Basantkumar, B. J. H. Stadler, W. P. Robbins and E. M. Summers, "Integration of Thin-Film Galfenol With MEMS Cantilevers for Magnetic Actuation," Magnetics, IEEE Transactions on, vol. 42, no. 10, pp. 3102-3104, 2006.

[12] A. E. Clark, J. B. Restorff, M. Wun-Fogle, T. A. Lograsso and D. L. Schlagel, "Magnetostrictive properties of body-centered cubic Fe-Ga and Fe-Ga-Al alloys.," Magnetics, IEEE Transactions on., vol. 36, no. 5, pp. 3238-3240, 2000.

[13] I. Chopra and J. Sirohi, Smart structures theory (Vol. 35)., Cambridge University Press, 2013.

[14] A. E. Clark, "Magnetostrictive rare earth-Fe 2 compounds," in Handbook of ferromagnetic materials, Elsevier, 1980, pp. 531-589.

[15] J. H. Yoo and A. B. Flatau, "A bending-mode galfenol electric power harvester," Journal of Intelligent Material Systems and Structures, vol. 23, no. 6, pp. 647-654, 2012.

[16] P. R. Downey, A. B. Flatau, P. D. McGary and B. J. Stadler, "Effect of magnetic field on the mechanical properties of magnetostrictive iron-gallium nanowires," Journal of Applied Physics, vol. 103, no. 7, p. 07D305, 2008. 
[17] B. Adolphi, J. McCord, M. Bertram, C. G. Oertel, U. Merkel, U. Marschner, C. Wenzel and W. J. Fischer, "Improvement of sputtered Galfenol thin films for sensor applications," Smart Materials and Structures, vol. 19, no. 5, p. 055013, 2010.

[18] T. Ueno, E. Summers, M. Wun-Fogle and T. Higuchi, "Micro-magnetostrictive vibrator using iron-gallium alloy," Sensors and Actuators A: Physical, vol. 148, no. 1, pp. 280-284, 2008.

[19] Z. G. Zhang, T. Ueno and T. Higuchi, "Development of a magnetostrictive linear motor for microrobots using Fe-Ga (Galfenol) alloys.," Magnetics, IEEE Transactions on, vol. 45, no. 10, pp. 4598-4600., 2009.

[20] P. D. McGary, L. Tan, J. Zou, B. J. Stadler, P. R. Downey and A. B. Flatau, "Magnetic nanowires for acoustic sensors," Journal of Applied Physics, vol. 99, no. 8, p. 08B310, 2006.

[21] H. R. Holmes, A. DeRouin, S. Wright, T. M. Riedemann, T. A. Lograsso, R. M. Rajachar and K. G. Ong, "Biodegradation and biocompatibility of mechanically active magnetoelastic materials," Smart Materials and Structures, vol. 23, no. 9, p. 095036, 2014.

[22] A. Sharma, Y. Zhu, M. Reddy, A. Hubel, R. Cobian, L. Tan and B.J. Stadler, "Steerable Nanobots for Diagnosis and Therapy," in Universe of Scales: From Nanotechnology to Cosmology, Springer International Publishing, 2014, pp. 179-189.

[23] E. C. Estrine, W. P. Robbins, M. M. Maqableh and B. J. Stadler, "Electrodeposition and characterization of magnetostrictive galfenol (FeGa) thin films for use in microelectromechanical systems.," Journal of Applied Physics, vol. 113, no. 17, p. 17A937, 2013.

[24] N. J. Hallab, K. J. Bundy, K. O'connor, R. Clark and R. L. Moses, "Cell adhesion to biomaterials: correlations between surface charge, surface roughness, adsorbed protein, and cell morphology," Journal of long-term effects of medical implants, vol. 5, no. 3, pp. 209-231, 1994.

[25] X. Wen, X. Wang and N. \& Zhang, "Microrough surface of metallic biomaterials: a literature review," Biomedical materials and engineering, vol. 6, no. 3, pp. 173-189, 1996.

[26] G. X. Wang, Y. Shen, H. Zhang, X. J. Quan and Q. S. Yu, "Influence of surface microroughness by plasma deposition and chemical erosion followed by $\mathrm{TiO} 2$ coating upon anticoagulation, hydrophilicity, and corrosion resistance of NiTi alloy stent.," Journal of Biomedical Materials Research Part A, vol. 85, no. 4, pp. 1096-1102, 2008.

[27] H. Liu and Z. L. Wang, "Etching silicon wafer without hydrofluoric acid.," Applied Physics Letters, vol. 87, no. 26, p. 261913, 2005.

[28] M. Takahashi and T. Shimatsu, "Soft magnetism of crystalline Fe based alloy sputtered films," IEEE Transactions on Magnetics, vol. 26, no. 5, pp. 1485-1490, 1990.

[29] E. Fernandez-Rosas, R. Gomez, E. Ibanez, L. Barrios, M. Duch, J. Esteve, C. Nogués and J. A. Plaza, 
"Intracellular polysilicon barcodes for cell tracking," Small, vol. 5, no. 21, pp. 2433-2439, 2009.

[30] T. Patiño, J. Soriano, E. Amirthalingam, S. Durán, A. González-Campo, M. Duch, E. Ibañez, L. Barrios, J. A. Plaza, L. Pérez-García and C. Nogués, "Polysilicon-chromium-gold intracellular chips for multi-functional biomedical applications," Nanoscale, vol. 8, no. 16, pp. 8773-8783, 2016.

[31] K. Butter, P. H. H. Bomans, P. M. Frederik, G. J. Vroege and A. P. Philipse, "Direct observation of dipolar chains in iron ferrofluids by cryogenic electron microscopy," Nature materials, vol. 2, no. 2, pp. 88-91, 2003.

[32] J. Xie, K. Chen and X. Chen, "Production, modification and bio-applications of magnetic nanoparticles gestated by magnetotactic bacteria," Nano research, vol. 2, no. 4, pp. 261-278, 2009.

[33] J. Dobson, "Remote control of cellular behaviour with magnetic nanoparticles.," Nature nanotechnology, vol. 3, no. 3, pp. 139-143, 2008.

[34] O. Felfoul, M. Mohammadi, S. Taherkhani, D. De Lanauze, Y. Z. Xu, D. Loghin, S. Essa, S. Jancik, D. Houle, M. Lafleur, L. Gaboury, M. Tabrizian, N. Kaou, M. Atkin, T. Vuong, G. Batist, N. Beauchemin, D. Radzioch and S. Martel, "Magneto-aerotactic bacteria deliver drug-containing nanoliposomes to tumour hypoxic regions," Nature Nanotechnology, 2016.

[35] T. Hogg, "Distributed control of microscopic robots in biomedical applications," in Advances in Applied SelfOrganizing Systems, London, Springer, 2013, pp. 179-208. 